\title{
Clinical Characteristics of Egyptian Patients with Inflammatory Bowel Diseases Infected with COVID-19: Tertiary Center Experience from Egypt
}

\author{
Ahmed Fouad Sherief, Mostafa Abd Alfattah Shamkh*, Mohamed Amin Sakr, \\ Waleed Hamed Abd Alaty, Ahmed Nagah Bassuny, Ibrahim Abdelhakim Ibrahim, \\ Safaa Ragab Askar, Shimaa Yousef Kamel, Heba Rashad, Mohamed Mahmoud Eltabbakh
}

Inflammatory Bowel Diseases Unit, Tropical Medicine Department, Faculty of Medicine, Ain Shams University, Cairo, Egypt

Email address:

masrawy_ainshams@yahoo.com (M. A. A. Shamkh)

${ }^{*}$ Corresponding author

\section{To cite this article:}

Ahmed Fouad Sherief, Mostafa Abd Alfattah Shamkh, Mohamed Amin Sakr, Waleed Hamed Abd Alaty, Ahmed Nagah Bassuny, Ibrahim Abdelhakim Ibrahim, Safaa Ragab Askar, Shimaa Yousef Kamel, Heba Rashad, Mohamed Mahmoud Eltabbakh. Clinical Characteristics of Egyptian Patients with Inflammatory Bowel Diseases Infected with COVID-19: Tertiary Center Experience from Egypt. American Journal of BioScience. Vol. 9, No. 2, 2021, pp. 47-54. doi: 10.11648/j.ajbio.20210902.13

Received: March 11, 2021; Accepted: March 22, 2021; Published: March 30, 2021

\begin{abstract}
During COVID-19 pandemic, inflammatory bowel disease patients were significantly worried about being at a higher risk of getting COVID-19 infection, the effect of their medications on the course of infection and the expected prognosis. This is a retrospective cohort study done in Our Inflammatory bowel disease unit, Tropical Medicine Department, Ain Shams University Hospitals, Cairo, Egypt. We retrospectively reviewed all our patients infected with COVID-19 (13 patients) during the period from March 2020 till mid-September 2020. Thirteen patients in our unit were infected with COVID-19. The mean age of infected patients was $39.92 \pm 11.16$ years. Most of them were females 11 (84.6\%). Most of them had ulcerative colitis (61.5\%) and only $38.5 \%$ had crohn's disease. Only six patients were admitted to isolation hospital, all of them were ulcerative colitis. The most common presenting symptoms were fever (84.6\%), cough (76.9\%) and diarrhea (61.5\%). Three of ulcerative colitis patients encountered disease exacerbation. All our infected patients had a good prognosis regarding their inflammatory bowel disease and COVID-19 course. COVID-19 infection in inflammatory bowel disease patients may carry a favorable outcome despite the vulnerability of those patients.
\end{abstract}

Keywords: Ulcerative Colitis, Crohn's Disease, COVID-19, Egypt

\section{Introduction}

Emergence of the newly discovered infectious severe acute respiratory syndrome (SARS co-v 2) virus was detected in China in December 2019. [1] The National Health Commission (NHC) of the People's Republic of China later announced that a novel coronavirus was responsible for the outbreak. The global attention was directed towards the new virus after its spread worldwide, followed by its declaration by the WHO as a global pandemic. [2] The number of new cases worldwide every day is rising reaching more than 81 million confirmed cases with about 1,772,222 deaths. Egypt till now encountered a slowly rising curve with about 132541 confirmed cases with 7405 deaths till the time of writing this manuscript. [3]

Beside the patients with comorbid diseases and elderly, patients with inflammatory bowel diseases (IBD) are theoretically at a higher risk for getting the infection based on type of immunosuppression medications and the severity of the disease. $[4,5]$

The COVID-19 pandemic has led to a very high need for hospitalization for symptomatic cases. This had its effect on patients suffering from diseases that require regular and continuous follow up including IBD patients with special consideration in treatment plans and follow up of those patients. [6] 
Different studies found a role for immunosuppression and corticosteroids in treating severe cases of COVID-19 patients by targeting the cytokine storm. This fact was approved and mentioned in national treatment protocols for management of severely and critically ill patients. [7, 8]

Immunosuppressive drugs and immunomodulators are the most used drugs in management of IBD. According to the previously given data, different worldwide studies were started to explain the pattern of the disease in such special group of patients.

Our current study is discussing the clinical characteristics of Egyptian patients with inflammatory bowel diseases infected with COVID-19 presented to our IBD unit with special emphasis on our protocol of management of IBD and COVID-19 infection.

\section{Materials and Methods}

\subsection{Study Design and Patients}

This is a retrospective cohort study. We retrospectively reviewed all our IBD patients infected with COVID-19 (13 patients) during the period from March 2020 till mid-September 2020 (who were on regular follow up with our IBD study group in tropical medicine department, Ain Shams University, Cairo, Egypt).

All included cases were diagnosed with COVID-19 by reverse transcriptase polymerase chain reaction assay (RT-PCR). The diagnosis was made in our triage unit in Ain Shams University Hospitals, Cairo, Egypt or in the near residence hospital of the patient. The follow up of the patient was adjusted by Teleconsultation after assessment by nearby specialist in case of patients from remote governorates.

All cases provided informed consent by either the patient himself or his/her legal guardian or Professional Legal Representative.

This study was conducted according to the Declaration of Helsinki 1975, as revised in 2000 and approved by the Faculty of Medicine, Ain shams University ethical committee.

\subsection{Data Collection and Assessment}

Patients' demographics, medical comorbidities, symptoms, initial laboratory investigations and oxygen support category, were extracted from the patients' medical records. The received drugs for management of COVID-19 beside any change in patient's regular IBD treatment were recorded. The need for hospitalization and the outcome of the disease regarding morbidity and mortality was reported.

According to the national protocol, IBD patients infected with COVID-19 were classified into mild, moderate and severe cases according to the need of hospital admission, oxygen requirements and laboratory findings in each patient. [9]

\section{Results}

\subsection{General Characteristics of Patients}

During the study period, the included patients (13 patients) with IBD who were diagnosed with COVID-19, were eight patients with ulcerative colitis (UC) and five patients with Crohn's disease (CD). All the patients had their COVID-19 diagnosis confirmed with positive nasopharyngeal swabs for SARS CoV-2. The baseline and demographic characteristics of the patients are shown in (Tables $1 \& 2$ ).

Overall, none of the patients suffered from previous chronic chest diseases or abnormalities, none of them had extraintestinal manifestations and only one patient had undergone total proctocolectomy with ileal pouch anal anastomosis (IPAA) for UC previously. Of the eight patients with $\mathrm{UC}$; only two had a severe form of UC, three had moderate disease severity while the remaining three had mild disease at their first presentation (according to Truelove and Witts criteria). Regarding the patients with $C D$, the mean $C D$ activity index was $382.40 \pm 104.32$ at their first presentation. Five of the patients in this study were steroid dependent but none were steroid resistant.

As for the concomitant IBD treatment of the patients, all patients with UC $(n=8)$ were receiving mesalamine. As for immunomodulators; there were 8 patients in this study who were maintained on azathioprine. Four of the patients in this study were on biological therapy; three on adalimumab and one on infliximab.

The most common presenting symptoms for the patients were (in descending order of frequency) fever (84.6\%), cough (76.9\%), diarrhea (61.5\%), dyspnea (53.9\%), anosmia (46.2\%), abdominal pain $(15.4 \%)$, bloody diarrhea and vomiting (both $7.7 \%$ ).

All the patients $(n=13)$ had undergone high resolution CT chest imaging with seven patients showing ground glass appearance and one patient showing opacities in the CT images while the rest $(n=5)$ had normal CT chest scans. Initial laboratory investigations for IBD patients infected with COVID-19 had been shown in Table 3.

Seven of our patients underwent home isolation while six patients needed isolation at a hospital ward, all six of them were on oxygen masks but fortunately no patient needed ICU admission. All the patients $(\mathrm{n}=13)$ received azithromycin and paracetamol, while four patients needed an additional antibiotic (third generation cephalosporin: $n=3$, meropenem: $\mathrm{n}=1$ ). Nine patients received hydroxychloroquine, while five patients were given steroids (oral prednisolone). Ten patients needed anticoagulation (prophylactic: $n=5$, therapeutic: $n=5$ ); where seven patients were given enoxaparin and three patients were on rivaroxaban. Nine patients received nitazoxanide.

Overall, 10 of the patients had a stable course regarding their IBD activity during the period of COVID-19 affection, while three had exacerbations. The mean time for hospital stay for the six patients who were admitted to hospital was 17.5 days. All the patients $(n=13)$ had fortunately achieved cure from COVID-19 with clinical remission and negative PCR for SARS CoV-2. 


\subsection{Degree of Severity of COVID-19 in IBD Patients}

Outcome in patients isolated at home and those admitted to hospital (Table 4):

Regarding the clinical picture and presentation, dyspnea and diarrhea were significantly higher in the patients admitted at hospital than in the group of patients treated at home. The level of CRP was significantly different between IBD patients with COVID-19 isolated at home and patients admitted to hospital, other than that the rest of the lab parameters were not widely different between both subsets of patients. The need for hospitalization was significantly higher in UC patients than CD patients, none of the five patients with $\mathrm{CD}$ in this study needed hospitalization.

\subsection{IBD Course During COVID-19}

In this study 10 patients had a stable course of IBD during their COVID-19 affection, while three patients suffered from concomitant exacerbation. Flares of IBD clearly occurred in a significantly higher proportion in patients with a more severe course of COVID-19 ( $\mathrm{P}=0.038)$. Clinically, abdominal pain occurred more frequently in COVID-19 patients with IBD exacerbation $(\mathrm{P}=0.005)$. Regarding their laboratory markers, there was no clear difference between both groups except for liver enzymes (AST \& ALT) which were clearly higher in COVID-19 patients suffering an IBD flare. Figure 1 summaries the management protocol of IBD patients infected with COVID -19 in our IBD unit.

Course of COVID-19: (Table 5)

When the patients were stratified according to the severity of COVID-19 course (mild, moderate \& severe), the total leukocytic count and the level of CRP were significantly different between the three groups $(\mathrm{P}=0.038$ for TLC \& $\mathrm{P}=0.031$ for $\mathrm{CRP})$. The other clinical and laboratory parameters were not significantly different across the three groups. Details of IBD cases infected with COVID-19 were summarized in Table 6.

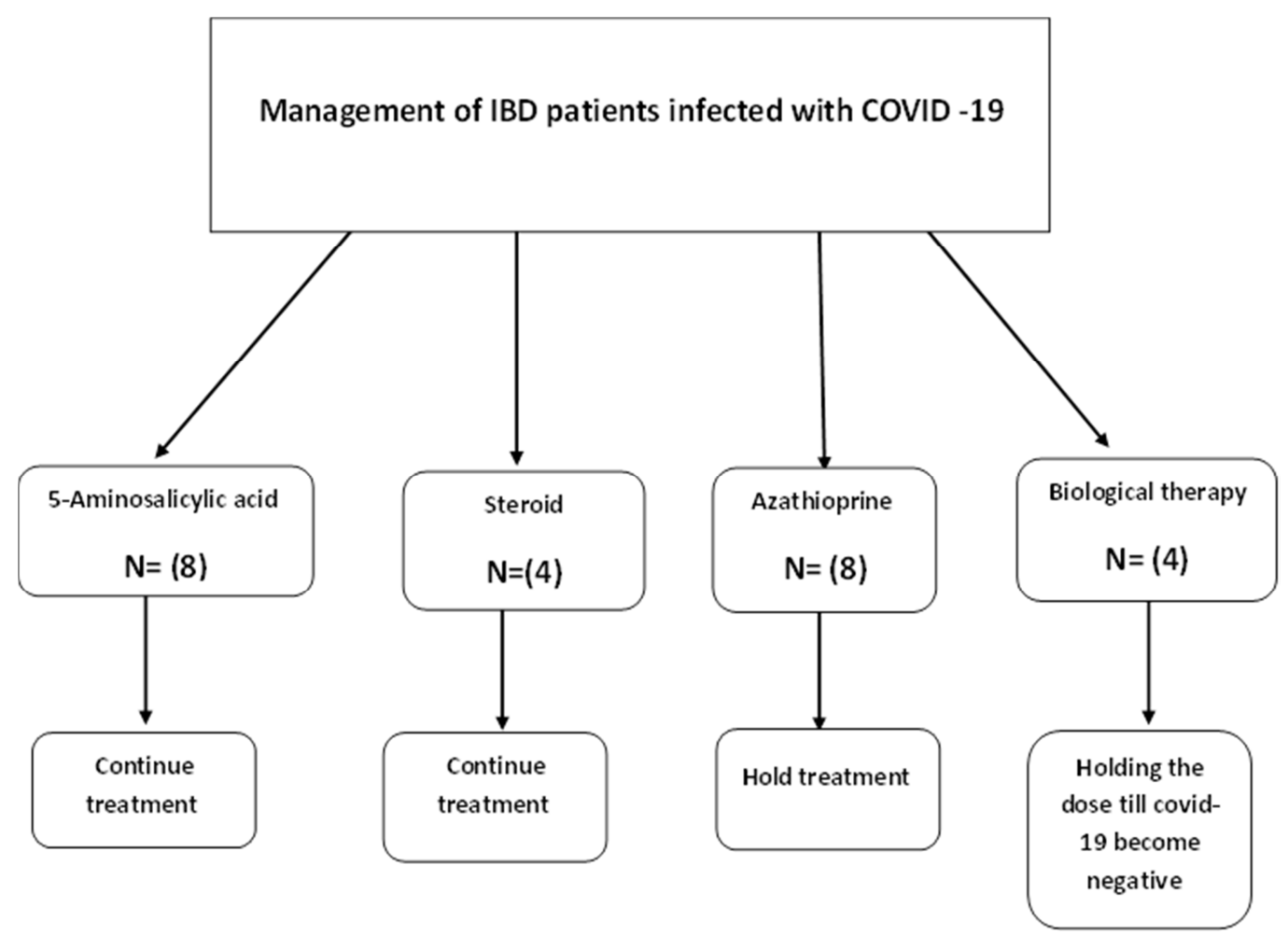

Figure 1. Management of IBD patients infected with COVID-19 in our IBD unit.

Table 1. Characteristics of IBD patients infected with COVID-19.

\begin{tabular}{lll}
\hline & & No. $=\mathbf{1 3}$ \\
\hline \multirow{2}{*}{ Age } & Mean \pm SD & $39.92 \pm 11.16$ \\
& Range & $20-65$ \\
Sex & Male & $2(15.4 \%)$ \\
& Female & $11(84.6 \%)$ \\
Type of disease & Ulcerative colitis & $8(61.5 \%)$ \\
& Crohns & $5(38.5 \%)$ \\
Marital status & Married & $8(61.5 \%)$ \\
& Single & $5(38.5 \%)$ \\
Smoking & Smoker & $0(0.0 \%)$ \\
Previous chest problems & Non-smoker & $13(100.0 \%)$ \\
\hline
\end{tabular}




\begin{tabular}{lll}
\hline & & No. $=\mathbf{1 3}$ \\
\hline & Negative & $13(100.0 \%)$ \\
Disease severity by Trulove and Witts criteria in & Mild & $3(37.5 \%)$ \\
ulcerative colitis at $1^{\text {st }}$ presentation & Moderate & $3(37.5 \%)$ \\
& Severe & $2(25.0 \%)$ \\
CDAI (Crohn's disease activity index) & Mean \pm SD & $382.40 \pm 104.32$ \\
& Range & $264-480$ \\
CDAI at $1^{\text {st }}$ presentation & Mild & $1(20.0 \%)$ \\
& Moderate & $2(40.0 \%)$ \\
Location of disease & Severe & $2(40.0 \%)$ \\
Ulcerative colitis & Rectal & $2(15.4 \%)$ \\
& Left & $1(7.7 \%)$ \\
Location of Crohn's disease & Pancolitis & $5(38.5 \%)$ \\
& Ileocolonic & $2(15.4 \%)$ \\
Dysplasia & Ileal & $3(23.1 \%)$ \\
& Skipped lesion colonic and small bowel & $0(0.0 \%)$ \\
Response to steroids: & Small bowel & $0(0.0 \%)$ \\
& No & $13(100.0 \%)$ \\
Extraintestinal & Not received in coarse of disease & $4(30.8 \%)$ \\
Previous surgery & Responding & $4(30.8 \%)$ \\
& Non responder & $0(0.0 \%)$ \\
& Steroid dependent & $5(38.5 \%)$ \\
& No & $13(100.0 \%)$ \\
& Yes & $1(7.7 \%)$ \\
& No & $12(92.3 \%)$ \\
\hline
\end{tabular}

Table 2. Maintenance therapy of our IBD cases infected with COVID-19.

\begin{tabular}{lll}
\hline & No. & \% \\
\hline 5-Aminosalisylic acid & 8 & $61.5 \%$ \\
Systemic & 7 & $53.8 \%$ \\
Topical & 0 & $0.0 \%$ \\
Both & 1 & $7.7 \%$ \\
Steroids & 4 & $30.8 \%$ \\
Oral & 4 & $30.8 \%$ \\
Topical & 0 & $0.0 \%$ \\
Azathioprine & 8 & $61.5 \%$ \\
Biological therapy & 4 & $30.8 \%$ \\
Infliximab & 1 & $7.7 \%$ \\
Adalimumab & 3 & $23.1 \%$ \\
\hline
\end{tabular}

Table 3. Initial laboratory investigations for IBD patients infected with COVID-19.

\begin{tabular}{lll}
\hline & & No. $\mathbf{1 3}$ \\
\hline \multirow{2}{*}{ oxygen saturation on room air } & Mean \pm SD & $95.46 \pm 3.31$ \\
Hemoglobin level & Range & $88-99$ \\
& Mean \pm SD & $11.55 \pm 1.72$ \\
Total leucocytic count & Range & $7.3-13.6$ \\
& Mean \pm SD & $6.54 \pm 1.99$ \\
Neutrophils & Range & $3.4-10.1$ \\
& Mean \pm SD & $4.28 \pm 1.78$ \\
Lymphocytes & Range & $2.2-8.3$ \\
& Mean \pm SD & $1.63 \pm 0.82$ \\
Neutrophil/Lymphocytic ratio & Range & $0.77-3.36$ \\
& Mean \pm SD & $3.12 \pm 1.78$ \\
Platelet count & Range & $0.73-7.55$ \\
& Mean \pm SD & $293.85 \pm 64.66$ \\
Aspartate aminotransferase & Range & $204-450$ \\
& Mean \pm SD & $31.00 \pm 14.33$ \\
Alaninie aminotransferase & Range & $11-66$ \\
D dimer & Mean \pm SD & $31.40 \pm 16.62$ \\
& Range & $15-78$ \\
Ferritin & Mean \pm SD & $594.08 \pm 365.36$ \\
C reactive protein & Range & $123-1292$ \\
& Mean \pm SD & $520.00 \pm 245.03$ \\
\hline
\end{tabular}



Infected with COVID-19: Tertiary Center Experience from Egypt

Table 4. Characteristics of IBD patients infected with COVID-19 who were admitted to the hospital.

\begin{tabular}{|c|c|c|c|c|c|c|}
\hline & & \multirow{2}{*}{$\begin{array}{l}\text { Home isolation } \\
7 \text { cases } \\
\end{array}$} & \multirow{2}{*}{$\begin{array}{l}\text { Hospital ward } \\
6 \text { cases }\end{array}$} & \multirow{2}{*}{ Test value } & \multirow{2}{*}{ P-value } & \multirow{2}{*}{ Sig. } \\
\hline & & & & & & \\
\hline \multirow{2}{*}{ Age } & Mean \pm SD & $34.71 \pm 8.46$ & $46.00 \pm 11.44$ & & & \\
\hline & Range & $20-47$ & $30-65$ & & & \\
\hline \multirow{2}{*}{ Type of disease } & Ulcerative colitis & $2(28.6 \%)$ & $6(100.0 \%)$ & \multirow{2}{*}{$6.964^{\mathrm{a}}$} & \multirow{2}{*}{$0.008^{*}$} & \multirow{2}{*}{ HS } \\
\hline & Crohns & $5(71.4 \%)$ & $0(0.0 \%)$ & & & \\
\hline \multirow{3}{*}{$\begin{array}{l}\text { Severity of } \\
\text { COVID-19 }\end{array}$} & Mild & $6(85.7 \%)$ & $1(16.7 \%)$ & \multirow{3}{*}{$6.533^{\mathrm{b}}$} & \multirow{3}{*}{$0.038^{*}$} & \multirow{3}{*}{$\mathrm{S}$} \\
\hline & Moderate & $1(14.3 \%)$ & $3(50.0 \%)$ & & & \\
\hline & Severe & $0(0.0 \%)$ & $2(33.3 \%)$ & & & \\
\hline \multirow{2}{*}{ Sex } & Male & $1(14.3 \%)$ & $1(16.7 \%)$ & \multirow{2}{*}{$0.014^{\mathrm{a}}$} & \multirow{2}{*}{0.906} & \multirow{2}{*}{ NS } \\
\hline & Female & $6(85.7 \%)$ & $5(83.3 \%)$ & & & \\
\hline \multicolumn{7}{|l|}{ Lab } \\
\hline Neutrophil/ & Mean \pm SD & $3.05 \pm 1.56$ & $3.21 \pm 2.16$ & \multirow{2}{*}{$-0.163^{c}$} & \multirow{2}{*}{0.874} & \multirow{2}{*}{ NS } \\
\hline Lymphocyte ratio & Range & $0.73-5.33$ & $1.8-7.55$ & & & \\
\hline D dimer & Mean \pm SD & $550.57 \pm 435.77$ & $644.83 \pm 294.55$ & $-0.448^{c}$ & 0.663 & NS \\
\hline \multirow{2}{*}{ Ferritin } & Mean \pm SD & $503.14 \pm 273.59$ & $539.67 \pm 231.10$ & $257^{\mathrm{c}}$ & & \\
\hline & Range & $235-965$ & $290-876$ & $-0.25 /$ & 0.802 & NS \\
\hline & Mean \pm SD & $36.14 \pm 30.96$ & $75.00 \pm 31.23$ & & & \\
\hline $\mathrm{C}$ reactive protein & Range & $6-96$ & $24-96$ & $-2.247^{c}$ & $0.046^{*}$ & $\mathrm{~S}$ \\
\hline & Range & $204-450$ & $242-340$ & & & \\
\hline oxygen saturation on & Mean \pm SD & $97.86 \pm 1.21$ & $92.67 \pm 2.66$ & $4655^{\mathrm{c}}$ & $0001 *$ & HS \\
\hline room air & Range & $96-99$ & $88-96$ & 4.035 & 0.001 & 110 \\
\hline Clinical picture & & & & & & \\
\hline Fever & & $5(71.4 \%)$ & $6(100.0 \%)$ & $2.026^{\mathrm{a}}$ & 0.155 & NS \\
\hline Cough & & $4(57.1 \%)$ & $6(100.0 \%)$ & $3.343^{\mathrm{a}}$ & 0.067 & NS \\
\hline Dyspnea & & $1(14.3 \%)$ & $6(100.0 \%)$ & $9.551^{\mathrm{a}}$ & $0.002 *$ & HS \\
\hline Anosmia & & $4(57.1 \%)$ & $2(33.3 \%)$ & $0.737^{\mathrm{a}}$ & 0.391 & NS \\
\hline abdominal pain & & $1(14.3 \%)$ & $1(16.7 \%)$ & $0.014^{\mathrm{a}}$ & 0.906 & NS \\
\hline Diarrhea & & $2(28.6 \%)$ & $6(100.0 \%)$ & $6.964^{\mathrm{a}}$ & $0.008 *$ & HS \\
\hline Bloody diarrhea & & $0(0.0 \%)$ & $1(16.7 \%)$ & $1.264^{\mathrm{a}}$ & 0.261 & NS \\
\hline 5-Amino salicylic acid & & $4(57.1 \%)$ & $4(66.7 \%)$ & $0.124^{\mathrm{a}}$ & 0.725 & NS \\
\hline Steroids & & $3(42.9 \%)$ & $1(16.7 \%)$ & $1.040^{\mathrm{a}}$ & 0.308 & NS \\
\hline Azathioprine & & $5(71.4 \%)$ & $3(50.0 \%)$ & $0.627^{\mathrm{a}}$ & 0.429 & NS \\
\hline Biologics & & $2(28.6 \%)$ & $2(33.3 \%)$ & $0.034^{\mathrm{a}}$ & 0.853 & NS \\
\hline
\end{tabular}

P-value $>0.05$ : Non significant (NS); P-value $<0.05$ : Significant (S); P-value $<0.01$ : highly significant (HS)

a: Chi-square test; ${ }^{\text {b: }}$ Mann Whitney test; ${ }^{\text {c}}$ : Independent t-test;

Table 5. Comparison between IBD cases infected with mild, moderate and severe COVID-19.

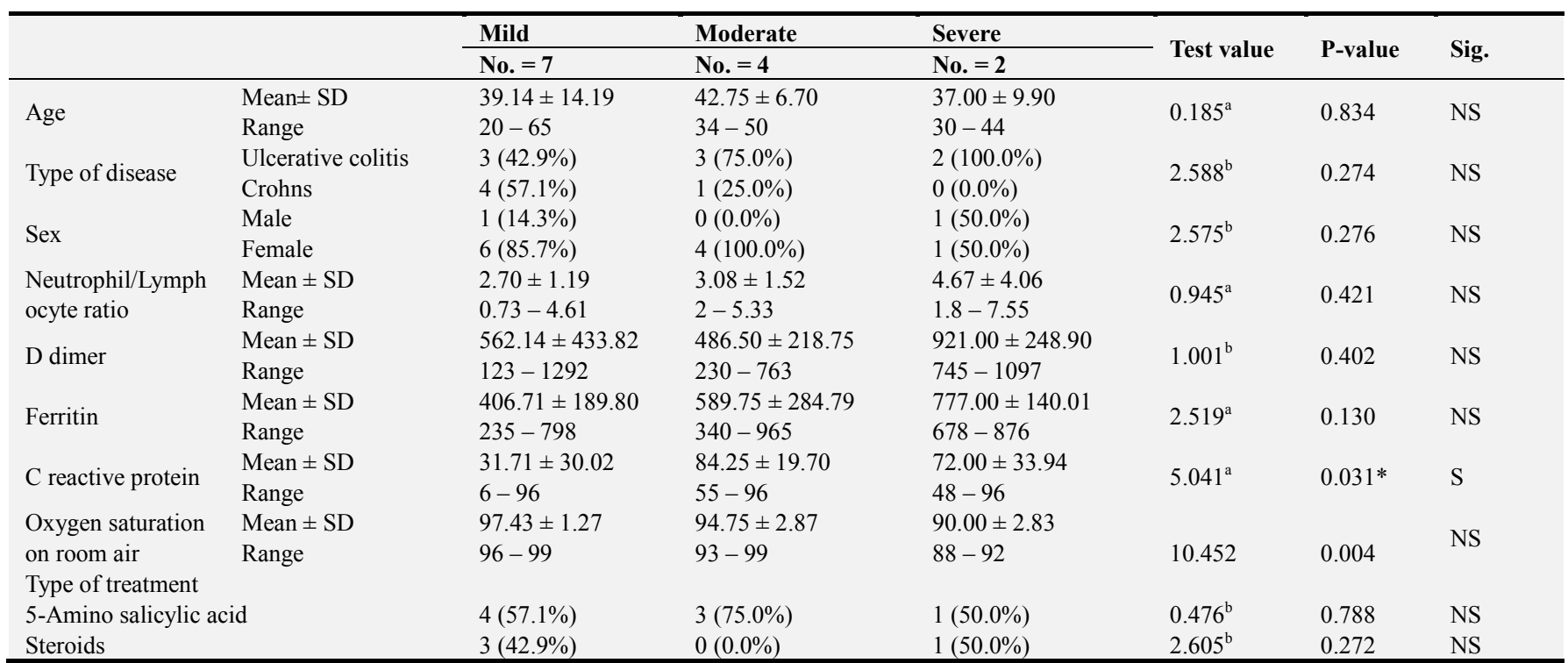




\begin{tabular}{|c|c|c|c|c|c|c|}
\hline & Mild & Moderate & Severe & & & \\
\hline & No. $=7$ & No. $=4$ & No. $=2$ & Test value & P-value & Sig. \\
\hline Azathioprine & $4(57.1 \%)$ & $2(50.0 \%)$ & $2(100.0 \%)$ & $1.532^{\mathrm{b}}$ & 0.465 & NS \\
\hline Biological therapy & $2(28.6 \%)$ & $1(25.0 \%)$ & $1(50.0 \%)$ & $0.426^{\mathrm{b}}$ & 0.808 & NS \\
\hline
\end{tabular}

P-value $>0.05$ : Non-significant (NS); P-value $<0.05$ : Significant (S); P-value $<0.01$ : highly significant (HS)

${ }^{\mathrm{a}}$ : One Way ANOVA test; ${ }^{\mathrm{b}}:$ Chi-square test;

Table 6. Summary of IBD cases infected with COVID.

\begin{tabular}{llllllll}
\hline & Type of IBD & Gender & Age & Location of disease in IBD & SO $_{2}$ on room air & $\begin{array}{l}\text { COVID-19 related GIT } \\
\text { symptoms }\end{array}$ & D- dimer level (ng/ml) \\
\hline Case 1 & UC & F & 42 & Pancolitis & 93 & Vomiting \& diarrhea & 230 \\
Case 2 & UC & F & 45 & Pancolitis & 94 & Diarrhea & 500 \\
Case 3 & CD & F & 20 & Ileocolonic & 97 & None & 740 \\
Case 4 & UC & F & 44 & Pancolitis & 92 & Diarrhea & 230 \\
Case 5 & CD & M & 30 & Ileal & 99 & None & 564 \\
Case 6 & CD & F & 34 & Ileal & 99 & None & 150 \\
Case 7 & CD & F & 34 & Ileal & 98 & None & 865 \\
Case 8 & UC & F & 50 & Pancolitis & 93 & Diarrhea & 340 \\
Case 9 & UC & F & 38 & Rectosigmoid & 99 & Diarrhea & 150 \\
Case 10 & UC & M & 30 & Rectosigmoid & 88 & Abdominal pain \& bloody diarrhea & 745 \\
Case 11 & CD & F & 40 & Ileocolonic & 96 & Abdominal pain & 1292 \\
Case 12 & UC & F & 47 & Pancolitis & 97 & Diarrhea & 189 \\
Case 13 & UC & F & 55 & Left sided & 93 & Diarrhea & 546
\end{tabular}

Table 6. Continue.

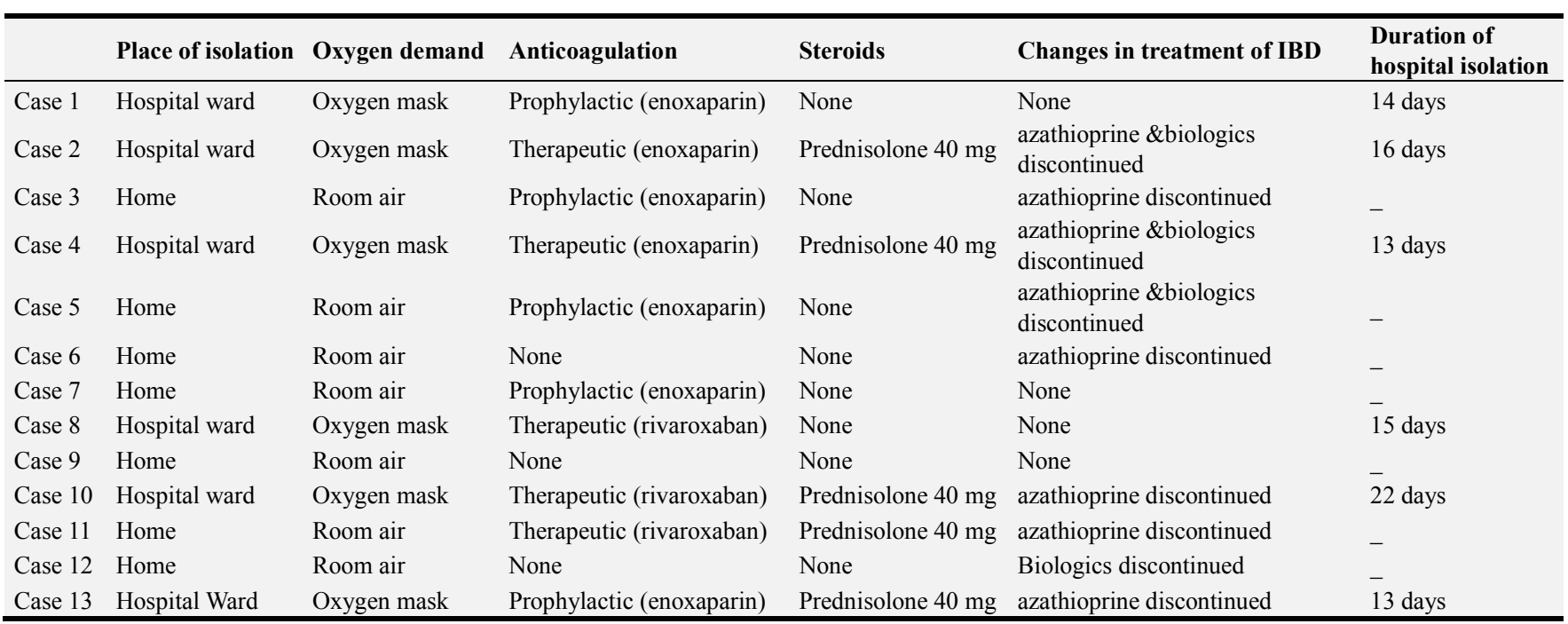

\section{Discussion}

IBD always seemed to be a rare disease in the Middle East and Northern Africa. There is no formal registry for patients with IBD in Egypt. The prevalence of IBD patients in Mediterranean countries was estimated at five per 100,000 in urban areas. [10] The low number of IBD cases is accompanied by a small number of studies reporting on IBD in our region.

Our IBD unit in Tropical Medicine Department, Ain Shams University Hospitals, Cairo, Egypt (one of the largest tertiary hospitals in Egypt) started its work in 2011. IBD patients are being followed up annually from all Egyptian governorates. [11] Patients with inflammatory bowel disease (IBD) have particular concerns for their risk of infection and management of their medical therapies.

On declaration of COVID-19 infections in Egypt at end of February 2020, we started to communicate with our patients through teleconsultations. A live weekly meeting was conducted online through Zoom application gathering all our IBD team discussing all newly diagnosed cases and follow-up of registered patients. [11]

The peak of COVID-19 in Egypt was in June till the beginning of July 2020 with a decrease in number of cases in the subsequent days. Fortunately, enough, only 13 IBD patients at our unit infected with COVID-19 in the period from March 2020 till the mid of September 2020. The most common presentations of COVID-19 were fever and respiratory manifestations. However, many patients now are presented by gastrointestinal manifestations like diarrhea which may reflect inoculation of the virus into the 
gastrointestinal (GI) tract and may be due to Angiotensin Converting Enzyme (ACE2) receptors expression in the intestines. [12] Most of our IBD patients infected with COVID-19 were presented with fever and respiratory manifestations. However, some of them presented with GI manifestations as diarrhea. Only few patients presented with abdominal pain and bloody diarrhea during their COVID-19 infection. Given the prevalence of non-specific digestive symptoms in COVID-19 cases especially in IBD patients, there are so many clinical implications that should be considered. Controlled IBD cases presented with diarrhea or other GI symptoms during COVID-19 pandemic should be tested for COVID-19 rather than be considered to have an exacerbation of the disease. [13]

Most of new guidelines are recommending continuing IBD treatment during COVID-19 pandemic. If the patient gets infection with COVID-19, he/she should withdraw corticosteroids, stop immunomodulators and postpone the usual dose of biological therapy. [13, 14, 15] Cytokine storm is one of the key features of COVID-19 infection. The use of potent anti-inflammatory drugs such as anti-tumor necrosis factor therapy may offer some protection for IBD patients and may be presented with milder disease if get infection with COVID-19. [16] In our cohort, four patients were on steroids, eight patients were on azathioprine and four patients were on anti-TNF therapy as maintenance therapy. According to our unit protocol [11], Azathioprine was held and anti-TNF therapy was postponed till resolution of infection. (Figure 1)

Six of our cohort were hospitalized either in our isolation hospital in Ain Shams University hospitals or in the nearest isolation hospital according to their residency. All patients (either hospitalized or not) improved and continued their IBD treatment after resolution of COVID-19 infection.

According to the Egyptian protocol for COVID-19 management [17], all our IBD patients received azithromycin and paracetamol. About $62 \%$ received hydroxychloroquine (after exclusion of cardiac problems and having an electrocardiogram). One of the interesting results in our cohort is the use of nitazoxanide in cases suffering from diarrhea with an excellent outcome. It is known that Nitazoxanide inhibits replication of a broad range of respiratory viruses in cell cultures, including SARS-CoV-2, and could be used as one line of management in the era of COVID-19 pandemic. [17]

\section{Conclusion}

IBD patients despite being a vulnerable group of patients may have a favorable outcome of COVID-19 infection. Despite the burden inflicted by the pandemic on the health system, the patients could still be followed up remotely by telephone. Patients with confirmed COVID-19 infection should contact their IBD team for proper management of their therapies and immunomodulators. Patients with a stable course of IBD should be kept on their medications without modification.

\section{Conflicts of Interest}

All the authors do not have any possible conflicts of interest.

\section{Data Availability Statement}

The data that support the findings of this study are available on request from the corresponding author. The data are not publicly available due to privacy and ethical restrictions.

\section{Funding}

All the authors declare that they didn't receive any funding for that research.

\section{References}

[1] Zhou P, Yang XL, Wang XG, Hu B, Zhang L, Zhang W, et al. A pneumonia outbreak associated with a new coronavirus of probable bat origin. Nature 2020; 579: 270-3.

[2] Wang C, Horby PW, Hayden FG, Gao GF. A novel coronavirus outbreak of global health concern [published correction appears in Lancet. 2020 Jan 29]. Lancet 2020; 395: 470-3.

[3] World Health Organization [Internet]. [Cited 2020 Dec 27]. Available from:

https://www.who.int/emergencies/diseases/novel-coronavirus2019/situation-reports/accessed 27/12/2020

[4] Beaugerie L, Kirchgesner J. Balancing Benefit vs Risk of Immunosuppressive Therapy for Individual Patients with Inflammatory Bowel Diseases. Clin Gastroenterol Hepatol 2019; 17: 370-9.

[5] Kirchgesner J, Lemaitre M, Carrat F, Zureik M, Carbonnel F, Dray-Spira R. Risk of Serious and Opportunistic Infections Associated with Treatment of Inflammatory Bowel Diseases. Gastroenterology 2018; 155: 337-46.

[6] Tezel A, Dökmeci G, Eskiocak M, Umit H, Soylu AR. Epidemiological features of ulcerative colitis in Trakya, Turkey. J Int Med Res 2003; 31: 141-8.

[7] Le RQ, Li L, Yuan W, Shord SS, Nie L, Habtemariam BA, et al. FDA approval summary: tocilizumab for treatment of chimeric antigen receptor $\mathrm{T}$ cell-induced severe or life-threatening cytokine release syndrome. The oncologist 2018; 23: 943.

[8] McCreary EK, Pogue JM. Coronavirus Disease 2019 Treatment: A Review of Early and Emerging Options. Open Forum Infect Dis 2020; 7: 105.

[9] Egyptian ministry of health and population. National protocol for management of COVID-19 patients. [Cited 2020 Dec 20]. Available from: http://www.mohp.gov.eg/JobsDetails.aspx?job_id=3061

[10] Mostafa EF, Metwally A, Hussein SA. Inflammatory bowel diseases prevalence in patients underwent colonoscopy in Zagazig University Hospitals. Afro-Egyptian Journal of Infectious and Endemic Diseases 2018; 8: 81-7. 
[11] Eltabbakh MM, Shamkh MAA, Bassuny AN, Abd Alaty WH, Sakr MA, Sherief AF. Inflammatory Bowel Diseases in Egypt During the COVID-19 Pandemic. Inflamm Bowel Dis 2020; 26: 1771-8.

[12] Pan L, Mu M, Yang P, Sun Y, Wang R, Yan J, et al. Clinical characteristics of COVID-19 patients with digestive symptoms in Hubei, China: a descriptive, cross-sectional, multicenter study. Am J Gastroenterol 2020; 115: 766-73.

[13] Rubin DT, Feuerstein JD, Wang AY, Cohen RD. AGA Clinical Practice Update on Management of Inflammatory Bowel Disease During the COVID-19 Pandemic: Expert Commentary. Gastroenterology 2020; 159: 350-7.

[14] Mao R, Liang J, Shen J, Ghosh S, Zhu LR, Yang H, et al. Implications of COVID-19 for patients with pre-existing digestive diseases. [published correction appears in Lancet Gastroenterol Hepatol. 2020 Jul; 5: e6]. Lancet Gastroenterol Hepatol 2020; 5: 426-8.

[15] Fiorino G, Allocca M, Furfaro F, Gilardi D, Zilli A, Radice S, et al. Inflammatory bowel disease care in the COVID-19 pandemic era: the Humanitas, Milan, experience. J Crohns Colitis 2020; 14: 1330-3.

[16] Allocca M, Fiorino G, Zallot C, Furfaro F, Gilardi D, Radice S, et al. Incidence and patterns of COVID-19 among inflammatory bowel disease patients from the Nancy and Milan cohorts. Clin Gastroenterol Hepatol 2020; 18: 2134-5.

[17] Martins-Filho PR, Barreto-Alves JA, Fakhouri R. Potential role for nitazoxanide in treating SARS-CoV-2 infection. Am J Physiol Lung Cell Mol Physiol 2020; 319: 35-6. 